\title{
Improved Seamless Cloning based Image In painting
}

\author{
Uppuretla Pavan Kumar, ${ }^{1}$ T.S.R Krishna Prasad ${ }^{2}$ \\ M.Tech Student, ${ }^{1}$ Associate Professor ${ }^{2}$ \\ ECE Department, Gudlavalleru engineering college, Krishna District \\ Andhra Pradesh, India
}

\begin{abstract}
Image in-painting is a restoration of image parts which are missing either accidentally or intentionally, and also image recovery is a research hot spot in the computer graphics and computer vision in the current. Two major inpainting techniques are introduced, those are PDE-based and Texture based algorithms. In PDE-based technique a conventional image matching techniques may be classified as either area based or feature based methods. In this paper, taking an input image and apply Pre-processing, After we get gray image and contour extraction algorithm is used to extract the contour features of damaged area, which determine the contour feature location. After that Matching algorithms play a key role in deciding correspondences between two image scenes. Area-based matching algorithm is used to search alternative matching area .Contour-based Similarity Distance function (CSD) is used to determine the most similar matching area. According to the damaged area and the matching area, the source cloning domains and target cloning domains of Seamless Cloning algorithm is improved. Using mean value coordinates to achieve regional pixel cloning from the source cloning domain to the target cloning domain. Finally, the improved SC algorithm is used to repair the image. Experimental results confirm the effectiveness of the proposed algorithm, and that the method can not only repair small-scale scratches, but also repair a large area damaged areas.
\end{abstract}

Keywords: Contour extraction; Image inpainting; Matching; Seamless cloning

\section{Introduction}

The filling-in of missing region in an image is known as Image Inpainting. Inpainting is the art of modifying an image or video in a form that is not easily detectable by an ordinary observer. Image Inpainting has become a fundamental area of research in image processing. The modification of images in a way that is non-detectable for an observer who does not know the original image is a practice as old as artistic creation itself. At present, the digital image inpainting techniques development focus on two research area, the first one is the image inpainting based on non-texture. In essence, it is an image inpainting algorithm of the Partial Differential Equation (PDE). This technique was firstly introduced image processing by Bertalmio and Bellester [20] and others in 2000 , and later Bertalmio also used third-order PDE simulate a smooth transfer process [19]; Chan-Shen used third-order PDE simulate Curvature-Driven Diffusion (CDD), and later to be proposed the Total Variation (TV) [17] model and so on.

PDE is designed to connect edges or isophotes (line of equal gray values). In Bertalmio et al. proposed an image inpainting algorithm based on PDE. Its algorithm will have good results if missed regions are small, but in large damaged regions it will take so long time and won't have good results. In Bertalmio et al [14]. use similarities between image processing and fluid dynamic topics, and he proposed an image inpainting algorithm using the Navier-Stokes equation. In authors try to minimize the TV-norm of reconstructed image in order to restore damaged pixels. Chan and Shen use energy functional involving the curvature of the level curves and tries to connect level curves in a smoothing fashion [16]. Masnou and Morel [21] in present a new model for image inpainting. They propose a simple but effective algorithm to connect level lines. Some works relate phase transition in supper conductors to image inpainting. Authors in propose an algorithm that propagates tangential and normal vectors into missed regions then it reconstructs damaged image by fitting it to these vectors. Telea [10] propose a fast matching method that can be considered as a PDE method which is faster and simpler to implement than other PDE based algorithms. All of above mentioned algorithms are very time consuming and have some problems with the damaged regions with a large size.

These methods belong to the first class image inpainting techniques, but they apply only to repair the cracks and some small-scale damaged in image. The other one is the image completion technology. At present, this type technology also includes two methods, one method is Bertalmio and others were made in 2003 based on image decomposition of the image inpainting techniques, whose main idea is to decompose the image into structural part and textures part, which structure part obtains the missing region by propagating linear structure into the target region via diffusion. They are inspired by the partial differential equations. Their drawback is that the diffusion process introduces some blur, which becomes noticeable when filling larger regions. Such methods 
are used by literature, but it used global search methods to lead to repairing time long, therefore, the above algorithm, remains to be further studied. Another method is first proposed to use exemplar -based texture synthesis techniques by Criminisi and others in 2003 [15], the main idea of this type algorithm is to select a pixel point to come from border of the damaged area, this point as the center, according to the image texture features [11], select the texture block of the appropriate size, and then search for the damaged area with the most similar texture matching block to replace the texture block [9]. Later, Harrison [18] also proposes a templatebased algorithm to remove the unwanted target, but this algorithm makes image line structure to become blurred. Drori and others propose a image completion algorithm based on fragments [13], the algorithm is the use of self-similarity principle, use a method of from coarse to fine to iterative approximation the regional of missing information, although we have had good inpainting results, but the algorithm use the global search strategy to ignore the image local similarity, affect the image inpainting quality. Although the literature gives a fast algorithm, it repairs the visual effects of the image to decrease correspondingly. The second category image inpainting techniques can repair the damaged large areas, but there are still some problems. In recent years, the mean value coordinates theory [12] and adaptive grid [8] have been proposed, a seamless cloning beginning to appear in the field of image reproduction and image fusion [7], although theory and algorithms are being developed. But it for the performance of image fusion to effectively overcome the short comings of wavelet analysis, for image inpainting provides new ideas and solutions. This paper is based on the above theory and algorithm, to propose a new image inpainting algorithm based on contour features and improved SC. The image inpainting algorithm flowchart is shown Figure 1.

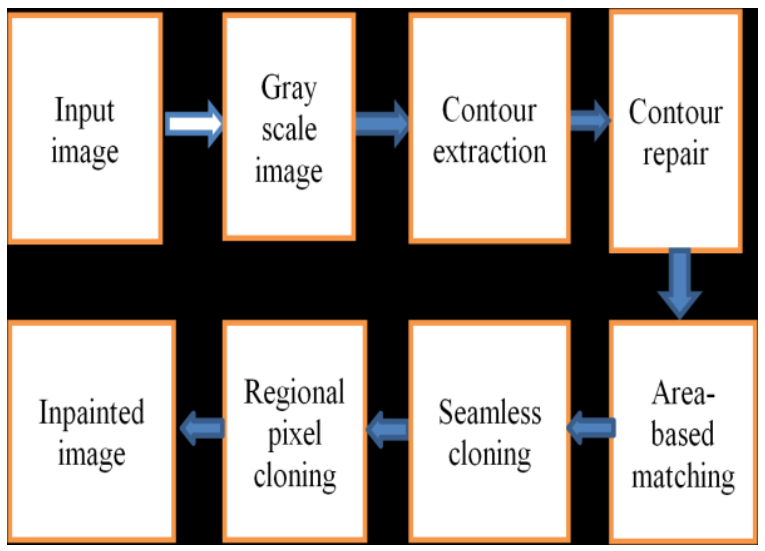

Figure 1.Image inpainting algorithm flowchart.

Image pre-processing, it is a sequence of operation process of remove certain specific targets in the inputting image ,image gray change, extract contour feature of the grayscale image, get contour features map. Image pre-processing can ensure the contour feature recognition and accuracy of the contour feature area-based matching, to improve the image inpainting quality.

First of all, the missing region is marked by the user and the accompanying target region whose center is that of the region is defined with a given thickness. Then the target region is compared to the all the possible region whose center is outside the target region in the sense of area-based matching [2]. Finally seamless cloning makes the boundary smooth.

Area-based matching, that is to use contour feature extraction algorithm to extract contour, to use a certain degree of matching strategy, to search for the best matching area.

Cloning algorithm [1] is provided the source cloning domain and target cloning domain to copy and fused by area-based matching algorithms, to get a full new image.

As shown above, the proposed method consists of two parts, that is, Area based matching and seamless cloning [5] which are explained in following subsections.

The organization of the paper is as follows. The existing matching algorithms is presented in section 2.The improved seamless cloning method is accessible in section 3.Chapter 4 deals with proposed method. Experimental results are represented in section 5 where a comparative study is done. Finally Chapter 6 describes conclusion and future scope.

\section{A. Composite Area Based Image Matching Method}

\section{Matching Algorithms}

The method is a composite technique where first the similarity measure between template window and search window is found by normalized cross correlation technique. Few best matches are selected for the template window from the search sub-windows, considering the largest normalized cross correlation coefficient. 
Further edge map is obtained for stereo image pair using canny edge detector. The matches for the template window are filtered using Hausdorff distance technique [3]. Further texture analysis of the same template window and selected search windows is the third measure to decide the accurate match. Texture analysis is done with the co-occurrence matrices which is a two dimensional histogram of the occurrence of pair of intensity value in a given spatial relationship. With this composite method dense point to point correspondence can be achieved with greater accuracy.

This method is tolerant to radiometric distortions and parallel processing of the three techniques will improve the speed.

\section{B. Band Matching}

The missing region is manually marked. In Figure: 2 show the white region is the marked missing region and the red contour is its boundary.

Accordingly the target band is formed with the given region and thickness. The target band is compared to all the possible source bands whose shape and thickness are the same as those of the target band but the center is outside the target region.

Among the candidates, the optimal band is chosen as the one with minimum SSD value.

$$
\mathrm{SSD}_{\mathrm{k}}=\sum_{\mathrm{i}=1}^{\mathrm{N}}\left(\left(\mathrm{R}_{\mathrm{i} k}^{\mathrm{S}}-\mathrm{R}_{\mathrm{i}}^{\mathrm{t}}\right)^{2}+\left(\mathrm{G}_{\mathrm{i} k}^{\mathrm{S}}-\mathrm{G}_{\mathrm{i}}^{\mathrm{t}}\right)^{2}+\left(\mathrm{B}_{\mathrm{i} k}^{\mathrm{S}}-\mathrm{B}_{\mathrm{i}}^{\mathrm{t}}\right)\right)
$$

Where $\mathrm{S}$ and $\mathrm{t}$ stands for the source and target bands respectively. $\mathrm{R}, \mathrm{G}$ and $\mathrm{B}$ mean the color channels. $\mathrm{k}$ is the index of a source band and $\mathrm{N}$ is the number of pixels belonging to a band.

The selected band region is then copied into the blank region. Figure 2 shows an example of band in-painting process.

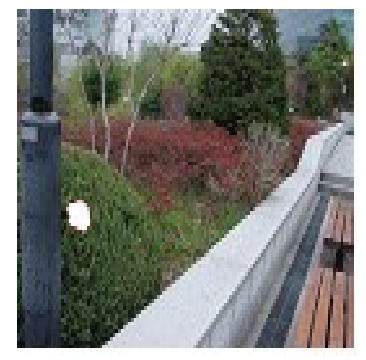

(a)

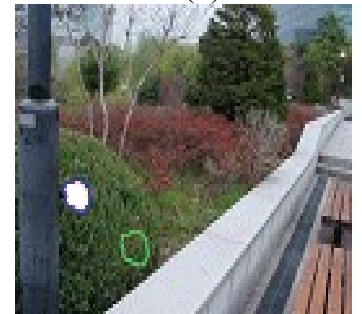

(c)

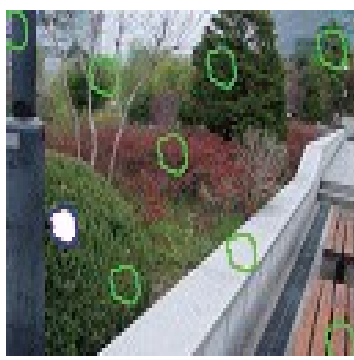

(b)

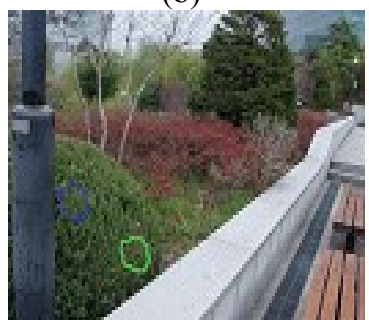

(d)

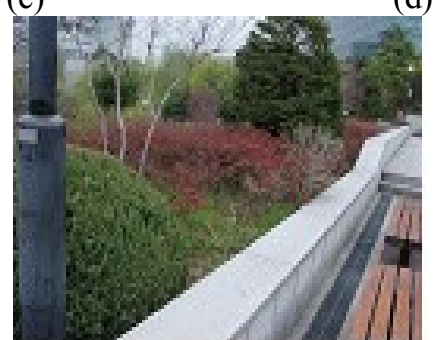

(e)

Fig. 2: An example of the band matching.

(a) The target region is marked. (b) The target band (blue) is compared to the source ones (green).

(c) The band with minimum difference (green) is found. (d) The blank region is filled with the image of the optimal band.

\section{Contour Extraction}

Contours and line drawings have been an important area in image data processing. In many applications, e.g., weather maps and geometric shapes, it is necessary to store and transmit large amounts of 
contours and line drawings and process the information by computers. Several approaches have been used to extract and encode the boundary points of contours and line drawings. Contour feature information is simple and feature point is little, using area-based matching based on the contour feature is conducive to shorten the matching time. The main purpose of the contour extraction is to make preparation for the area-based matching. The essence of the contour extraction [4] is that the contour feature image contains only 0 and 1 two kinds gray values based on the grayscale images, to use the 256 X 256 image as an object, the contour features image is a binary matrix. The contour extraction feature algorithm steps are as follows:

(1) Scan grayscale images after the image pre-processing, to detect the gray-level value of grayscale image.

(2) Binary image is established. The getting image can be constructed that pixels above the threshold are foreground (" 1 ") and below the threshold are background (" 0 ").Binary images are images whose pixels have only two possible intensity values. They are normally displayed as black and white. Numerically, the two values are used 0 for black and 1 for white. Which received two value matrix, according to two matrices can be established the binary image.

(3) Contour extraction of the damaged area can create the mask is established. According to received binary image to establish the mean value coordinates, and in accordance with certain rules to determine the boundaries of the region, automatically distinguish between contour and regions, so as to establish contour feature of the masked area.

Contour feature extraction results shown in Figure 3.

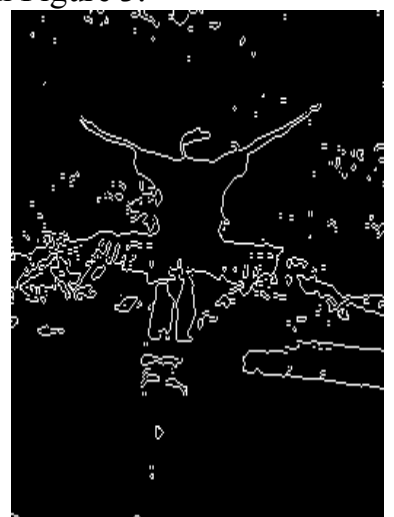

Figure 3: Contour feature extraction

\section{Contour Extraction feature depends on Area matching algorithm}

The purpose of area-based matching algorithm is to select matching area of the appropriate size with the contour feature of the image damaged area, and then search the best matching area to fill the damaged area. using contour-based feature similarity distance function (CSD) to determine the best matching area, set the contour region of the damaged image as the target cloning domain $\mathrm{A}$, its matching region as the source cloning domain B, and then using Improved Seamless Cloning algorithm to achieve the image inpainting. The main steps of area-based matching algorithm based on contour feature as follows:

(1) Contour extraction algorithm is used to extract contour feature, and through the mean value coordinates to establish the location coordinates and size of the damaged area (i.e. to be filled region).

(2) Search for matching area and identify alternative matching area. Selected to be filled area surrounding a pixel as the center, in turn pushed out, the matching pixels can change with the different shapes of to be filled region edges, until meet the size of be filled region. According to this search strategy will have n matching area, and then matching area as an alternative matching area of to be filled area.

(3) Alternative matching area is determined. Determine the one-to-many alternative matching area; search the global image to match, and using CSD to determine the alternative matching area. Provide to judging basis of to be filled area and matching area whether the best, using formula (2) to judge it.

$$
\delta=\sqrt{\frac{1}{n^{2}} \sum_{n}\left[\left(I_{R}^{A}-I_{R}^{B}\right)^{2}+\left(I_{G}^{A}-I_{G}^{B}\right)^{2}+\left(I_{B}^{A}-I_{B}^{B}\right)^{2}\right]}
$$

Where, $\mathrm{n}$ is the total number of pixels in the to be filled area, I is the pixel brightness value, $\mathrm{R}, \mathrm{G}$ and $\mathrm{B}$ representing respectively the three color components, the A and B respectively are to be filled area and alternative matching area.

In the $\mathrm{n}$ alternative matching area, select the minimum $\delta$ in the above calculation results, it corresponds the matching area $\mathrm{B}$ is the best match of to be filled area $\mathrm{A}$.

The mean value coordinates is used to determine location coordinates [6] of the matching area after determine the alternative matching area. 
Area-based matching method based on contour feature, make binary image contour as the feature, the amount of feature information significantly reduced, it will help shorten the matching time, to achieve real- time processing. The similarity distance function based on contour feature full play the characteristics of gray-scale images, avoid the links of set the threshold according to experience, lead to method more intuitive, fast.

\section{Improved Seamless Cloning Method}

The principle of traditional SC algorithm is that using the target cloning domain of an image A and the source cloning domains of the other image B to copy fusion, use two images to achieve the image inpainting, while this paper algorithm improvements is to use only a damaged image to achieve image inpainting.

\section{A. Resolved restoration area}

Area-based matching algorithm based on contour feature is used to determine the damaged area A and the matching area B in a to be repaired image, set the damaged area $\mathrm{A}$ as the target cloning domain, set matching area $\mathrm{B}$ as the source cloning domain, after determine the source cloning domain and the target cloning domain, using the improved mean value seamless cloning algorithm to achieve cloning, so that $\mathrm{A}=\mathrm{B}$, and then fusion methods of mean value seamless cloning algorithm to achieve the regional A seamless repair.

\section{B. Use Seamless cloning algorithms to image inpainting}

Let $\mathrm{B} \subset \mathrm{R}^{2}$ be the source cloning domain of the image $\mathrm{C}$ and $\mathrm{A} \subset \mathrm{R}^{2}$ be the target cloning domain of the image C. We can remember as follows:

$$
\mathrm{g}: \mathrm{A} \rightarrow \mathrm{R}, \mathrm{f} *: \mathrm{B} \rightarrow \mathrm{R} \text {. }
$$

Let $\mathrm{P}_{\mathrm{B}} \subset \mathrm{B}$ is the source cloning domain patches, the aim of seamless cloning is cloned $\mathrm{P}_{\mathrm{B}}$ into the target cloning domain $\mathrm{P}_{\mathrm{A}} \subset \mathrm{A}$.

Seamless cloning essentially computes a function: $\mathrm{PA} \rightarrow \mathrm{R}$, and solving the Poisson equation:

$\Delta \mathrm{f}=\operatorname{Div} \nabla \mathrm{gw} /$ Dirichlet boundary conditions:

$\mathrm{f} \mid \partial \mathrm{pA}=\mathrm{f} *$

In other words, seamless cloning seeks a function $\mathrm{f}$ that agrees with the target domain $\partial \mathrm{p}_{\mathrm{A}}$, whose gradient field is as close as possible to that of the source image $\mathrm{g}$.

Solving the above Poisson equation is equivalent to solving the Laplace equation:

$\Delta \mathrm{f}^{\prime}=0 \mathrm{w} /$ Dirichlet boundary conditions:

Consider a point $\mathrm{x} \in \mathrm{p}_{\mathrm{A}}$ with boundary

$$
\mathrm{f}^{\prime} \mid \partial \mathrm{pA}=\mathrm{f} *-\mathrm{g}
$$

$$
\partial \mathrm{pA}=(\mathrm{p} 0, \mathrm{p} 1, \mathrm{p} 2 \ldots \ldots \mathrm{pm}=\mathrm{p} 0) .
$$

The mean - value interpolant obtaining the values $\mathrm{f} *(\mathrm{pi})-\mathrm{g}(\mathrm{pi})$ at the boundary $\mid \partial \mathrm{p}_{\mathrm{A}}$ is given at point $\mathrm{x}$ by :

Where:

$$
\mathrm{r}(\mathrm{x})=\sum_{\mathrm{i}=0}^{\mathrm{m}-1} \lambda \mathrm{i}(\mathrm{x})(\mathrm{f} *(\mathrm{pi})-\mathrm{g}(\mathrm{pi}))
$$

$$
\begin{gathered}
\lambda \mathrm{i}(\mathrm{x})=\mathrm{wi} / \sum_{\mathrm{i}=0}^{\mathrm{m}} \mathrm{wj}, \mathrm{i}=0, \ldots, \mathrm{m}-1 ; \\
\text { wi }=(\tan (\mathrm{ai}-1 / 2)+\tan (\mathrm{ai} / 2)) /\|\mathrm{pi}-\mathrm{x}\|
\end{gathered}
$$

The result of mean value cloning is then given, similarlyto eq. (4), by

$$
f(x)=g(x)+r(x)
$$

MVC Seamless Cloning Algorithm:

1: \{pre-processing stage\}

2: for each pixel $\mathrm{x} \in \mathrm{p}_{\mathrm{B}}$ do

3: $\left\{\right.$ compute the mean-value coordinates $\lambda \mathrm{i}(\mathrm{x})$ and $\partial \mathrm{p}_{\mathrm{B}}$ \}

$4: \lambda 0(\mathrm{x}), \ldots ., \lambda \mathrm{m}-1(\mathrm{x})=\mathrm{MVSC}\left(\mathrm{x}, \mathrm{y}, \partial \mathrm{p}_{\mathrm{B}}\right) / /$ Mean coordinates of the calculation and storage

5: end for

6: for each new $\mathrm{p}_{\mathrm{A}} \mathrm{do}$

7: $\{$ compute the differences along the boundary\}

8: for $\partial \mathrm{p}_{\mathrm{i}}$ each vertex $\mathrm{p}_{\mathrm{i}}$ do

$9: \mathrm{h}=\mathrm{f} *(\mathrm{pi}-\mathrm{g}(\mathrm{pi})$

10: end for

11: for, $\partial \mathrm{p}_{\mathrm{A}}$ each vertex $\mathrm{x} \in \mathrm{P}_{\mathrm{A}}$ do

12: $\{$ evaluate the mean-value interpolant at $x$ \}

13: $r(x)=\sum_{i=0}^{m-1} \lambda i(x) . h / /$ Assignment

14: $f(x)=g(x)+r(x)$

15: end for

16: end for 
At last, seamless cloning algorithm is used to complete the image inpainting.

\section{Improved Seamless Cloning Based Image Inpainting}

In this paper area-based matching method based on contour feature is used, the method is good and is basis improved Seamless Cloning algorithm. SC algorithm is achieved by the cloning between the source cloning domains and the target cloning domains, the real repair seamlessly.

Algorithm steps are as follows:

(1) Input image and pre-processing, to get gray-scale image, Remove the specific goals in the inputting image, as well as gray changes.

(2) Extract the contour features of gray-scale image using Contour extraction algorithm, to determine the repaired region and contour in the binary image.

(3) Mean value coordinates is established, accurate determine the location coordinates and size of to be repaired contour feature region.

(4) Area-based matching algorithm based on contour feature is used to search alternative matching area, and use the formula (3) to determine the alternative matching area.

(5) To determine transformation parameters between the damaged area and the matching area, the least square method is used and provides the basis for the next phase the mean value seamless cloning.

(6) According to the information of the damaged area and match area are provided by area-based matching algorithms to determine respectively target cloning domain and the source cloning domain in SC.

(7) Mean value coordinates is used to achieve regional pixel to clone from the source cloning domain to the target cloning domain.

(8) Finally, this paper improved SC algorithm is used to achieve image inpainting to get the whole image.

\section{Experimental Results}

In order to verify the effectiveness of image inpainting algorithm which based on contour features and improved SC in this paper, we use three group experiments and three natural scene images with different level damage. As shown in Figure 4, three images (a), (b) and (c) respectively exists bungee image, tree, and writing text and as the inputting image. The three images (a), (b) and (c) in figure 5respectively is the result figure after removing specific target. We use these images to extract the contour feature, and then determine the damaged area contours. Figure 6 (a), (b) and (c) three images use traditional methods to repair the whole image, which is based on PDE, although it is able to complete the image inpainting, but after compare with the original image, to find the repaired image exist white spots. Figure 6 (d), (e) and (t) three images were adopted this paper algorithms to repair the image, compare with the original image and the traditional methods repaired image, to find image does not exist white spots. Obviously, by comparing this paper algorithm with the traditional method, this paper algorithm not only achieve good results in repairing small scale and large-area damaged region, but also provide a very good solution to the white spots and other issues which are left by the traditional method to repair damaged images.

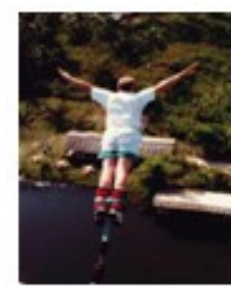

(a)

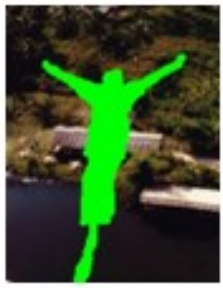

(a)

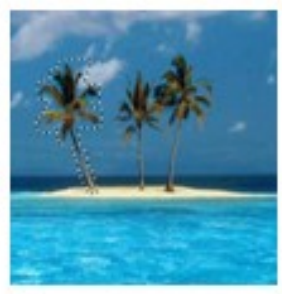

(b)

Figure 4.damaged image

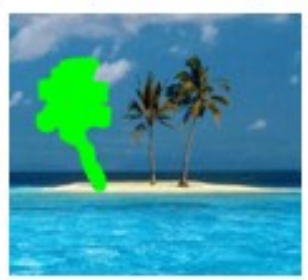

(b)

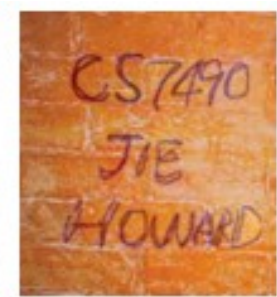

(c)

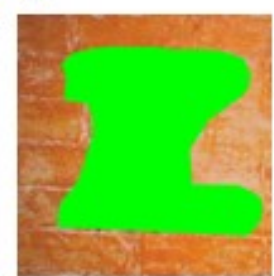

(c)

Figure 5.remove target image 


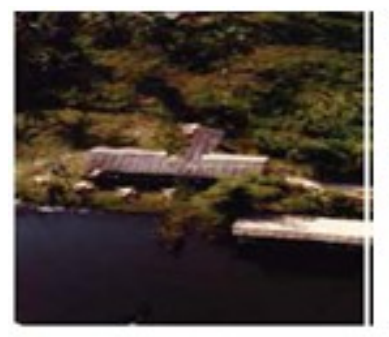

(a)

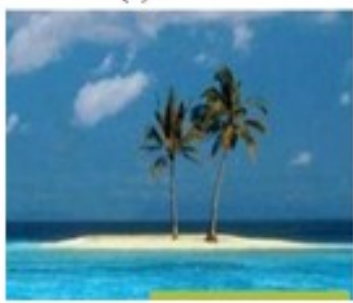

(b)

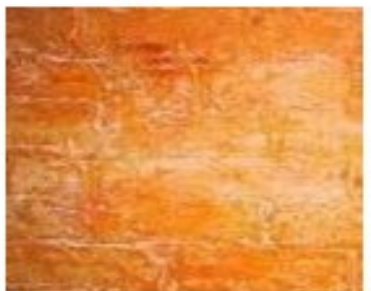

(d)

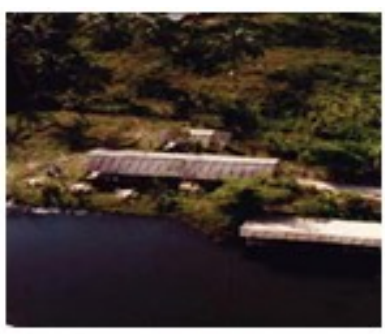

(d)

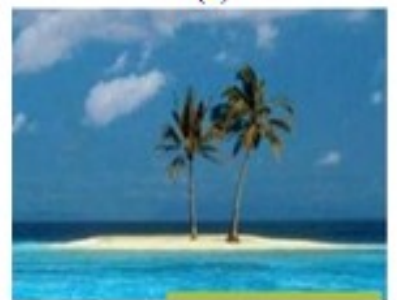

(e)

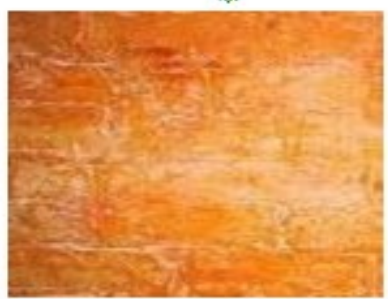

(f)

Figure 6.(a) - (c) image in painting based on $\mathrm{PDE}$

(d) - (f) this paper method

Experimental results show that this paper algorithm not only can repair small-scale damaged digital images, but also can fill and repair a large pieces of blank space on the image, and supply a very good solution to white spots and other issues left by the traditional method, achieve a complete smooth and no-slit image inpainting and improve the image inpainting quality.

\section{Conclusion And Future Scope}

The existing image completion algorithms are time consuming, and the results are not satisfactory. This paper presents an image inpainting algorithm based on contour features and improved SC. It not only make the damaged area and the matching area match exactly, but also achieve to repair the image small spots, scratches and the wide spread damaged region perfectly. Region matching method based on contour feature is the current research focus, but matching algorithm complexity is higher. Image cloning fusion based on Seamless Cloning is a new, rapid cloning and fusion method; it can quickly achieve the perfect image inpainting after using the relationship image of contour features area-matching. Looking for the future, image inpainting techniques should improve the matching speed, accuracy inpainting quality and other aspects of the damaged area.

\section{Acknowledgements}

The authors place on record their thanks to the authorities of Gudlavalleru Engineering College, A.P for the facilities they provided.

\section{References}

[1]. Shrilaxmi Deshpande \&Shalini Bhatia. "Image Inpainting Using Cloning Algorithms" [J] International Journal for Image Processing (IJIP), Volume (6): Issue (6): 2012.

[2]. JyotiJoglekar, Shirish S. Gedam. “Area Based Image Matching Methods”[J].International Journal of Emerging Technology and Advanced Engineering. ISSN 2250-2459, Volume 2, Issue 1, January 2012.

[3]. Joglekar J. V, Gedam S. S., Area Based Image Matching Technique using Haudorff distance and Texture analysis, ISPRS WG III/4 International conference PIA11, Technical University of Munchen, Germany, 2011.

[4]. Panagiotis Gioannis. "Automatic Contour Extraction from 2D Images" Applied Medical Informatics Original Research, Vol.28, No.1 / 2011. pp: 9-15.

[5]. Zhen Hua, Yewei Li \&Jinjiang Li. "Image Inpainting Algorithm based on Contour features and Improved MVSC" [J] International Conference on Computer Design And Applications (ICCDA), Volume (1) ,2010. Pp-212-216

[6]. FarbmanZeev, Hoffer Gil, LipmanYaron, Cohen-Or Daniel, Lischinski Dani."Coordinates for Instant Image Cloning". ACM Transactions on Graphics - TOG, 07300301, 2009, 28: 67-67.

[7]. Subin Lee and YongduekSeo. "Image In-painting by Band Matching and Seamless Cloning" [J]. Korea Science and Engineering Foundation (KOSEF) Appl.Math, 2006, pp-364-369. 
[8]. JU. T, SCHAEFER. S, WARREN. J. 2005. "Mean value coordinates for closed triangular meshes". ACM Trans. Graph. 24, 3, 561 566.

[9]. Wen Li, David Zhang, Zhiyong Liu and XiangzhenQiao, "Fast Block-Based Image Restoration Employing the Improved Best Neighborhood matching approach", IEEE Transaction On System, Man And Cybernetics - Part A Systems And Humans, Vol. 35, No.4, July 2005

[10]. A. Telea, "An image inpainting technique based on the fast matching method",journal of graphics tools, vol.9,no. 1, acm press 2004

[11]. Harald G. "A combined PDE and texture synthesis approach to inpainting" [A]. In: Proceedings of 8th European Conference on Computer Vision [C], Prague, Czech Republic, 2004 : 214-224.

[12]. Floater, M. S . 2003. "Mean value coordinates". Comput.Aided Geom. Des. 20, I, 19-27.

[13]. Drori I, Daniel C 0, Hezy Y. "Fragment based image completion" [J]. ACM Transactions on Graphics, 2003, 22 (3): 303 -312

[14]. Bertalmio M, Vese L, Sapiro G, eta!."Simultaneous texture and structure image inpainting" [1]. IEEE Transactions on Image Processing, 2003, 12 (8): 882-889.

[15]. C. P. Perez, Toyama K. "Region filling and object removal by Exemplar- based image inpainting[j]." IEEE Trans. Image Processing, 2003, 13(9). Page: 1200-1212.

[16]. T. Chan, J. Shen, "Mathematical models for local nontexture inpainting," SIAM J. Appl, Math., vol. 62, no. 3, pp. 1019-1043, 2001.

[17]. Chan T F, Shen J H. "Non-Texture Inpainting by Curvature - Driven Diffusions CDD" [J]. J. Visual Comm. Image Rep, 2001, 12(4): $436-449$

[18]. Harrison P. "A nonhierarchical procedure for resynthesis of complex texture" [ A]. In: Proceedings of 9th International Conference on Central Europe Computer Graphics, Visualization, and Computer Vision[C/OL], Plzen, Czech Republic, Feb. 2001.

[19]. Bertalmio M, Sapiro G, Caselles V, eta!." Image Inpainting" [C]II International Conference on Computer Graphics and Interactive Techniques, New York: ACM Press, 2000:417-424.

[20]. Bertalmio M.; Sapiro G.; V. Caselles et al; "Image Inpainting" In Kurt Akeley, editor, Siggraph 2000, Computer Graphics Proceedings. ACM Addison Wesley Longman, 2000. Page: 417-424.

[21]. S. Masnou, J. M. Morel, "Level lines based disocclusion," proceedings of the 1998 IEEE International conference of image processing, pp. 259-263. 